\title{
Systemic Service Design: Aligning Value and Implementation
}

\author{
Arash Golnam, Gil Regev, Julien Ramboz, Philippe Laprade and Alain Wegmann \\ Ecole Polytechnique Fédérale de Lausanne (EPFL) \\ School of Computer and Communication Sciences \\ CH-1015 Lausanne, Switzerland \\ \{arash.golnam, gil.regev, julien.ramboz, philippe.laprade, alain.wegmann\}@epfl.ch
}

\begin{abstract}
The promise of service orientation is that it enables an organization to prosper by delivering continuous value to customers. This prosperity is of strategic value to the organization. There is value in service orientation for both the organization and its customers. We call these two values, customer value and strategic value. When designing a service it is necessary to align both value propositions with the service building blocks. We propose to use a systemic method where whole and composite reasoning are interleaved on both the organizational and functional dimensions. We begin by producing an as-is model that describes how customer value is delivered by a set of actors and the responsibility of each actor. Improvement opportunities are identified in terms of customer value and strategic value to the organization. A to-be model is built that specifies the new interaction between actors and their new responsibilities. The method is illustrated with an example.
\end{abstract}

\section{Introduction}

The IT Infrastructure Library (ITIL) defines the concept of service as, "a means of delivering value to customers by facilitating outcomes customers want to achieve without the ownership of specific costs and risks" [1]. This definition can be split in two parts: 1) a service delivers value to customers by providing them with the outcomes that they desire. 2) The service relieves the customers from dealing with the cost and risks associated with the outcomes they desire. We call the first part the "service offering" and the second part the "service implementation". The ITIL definition reminds us that customers often care more about the value they derive from a service than the way the service is implemented. In most cases a service is offered as a black box, without customers knowing how it is implemented. The service provider, however, must make sure that the implementation is capable of delivering the value expected by customers. Service design is the act of aligning the value (the black box view as seen by customers) with the implementation (the white box view as seen by the service provider). The service provider provides the service to customers for a reason. It benefits directly and indirectly from the relationship with customers. Direct benefits include service payments made by customers. Indirect benefits can be the growth of the customer base leading to more customer payments. Hence, the 
service provider also finds value in the service. We therefore distinguish between customer value (the value delivered to the customer) and strategic value (the value to the service provider). The service offering includes both these value propositions.

In this paper, we show how to the Systemic Enterprise Architecture Modeling (SEAM) can be used to design the service offering and the service implementation while capturing the customer and strategic value. SEAM designates a family of Enterprise Architecture methods. In this paper, we describe a version of SEAM for service design. For sake of simplicity we call it SEAM rather than SEAM for Service Design. Each SEAM method includes a design process and an enterprise model. The foundations of SEAM are in General Systems Thinking (GST) [2] and in RM-ODP [3]. GST is the study of principles that are applicable to any kind of system (e.g. business system or IT system). RM-ODP is a software engineering ISO standard that provides solid definitions for the SEAM concepts. SEAM is rigorously defined based on these systemic and software engineering concepts (e.g. object, state, behavior). SEAM federates multiple modeling techniques (such as discrete behavior, goals or quantitative models). SEAM has been applied for teaching [4] and consulting [5] since 2001. Prior applications of SEAM to service design have been published in [6] and [7].

The SEAM design process has three phases as illustrated in Fig. 1. First, we analyze the service offering and implementation as-is to identify how well the service offering is aligned with the service provider's strategic value. We then identify the service improvement opportunities. This leads to the redesign of the service offering and implementation. The dashed arrow in Fig. 1 suggests that the method can be applied more than once for continuous improvement.

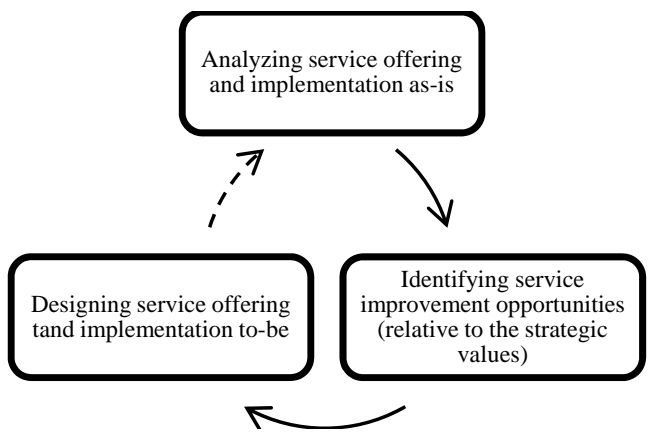

Fig. 1. Phases in design process

The SEAM enterprise model for designing services conceptualizes the service provider and its environment as a hierarchy of systems that includes value segments, value networks, companies, IT systems and employees. Fig. 2 illustrates this hierarchy of systems.

We define a system as a group of entities that interact and that we can consider as a whole (not showing the system's components, also known as black box) as well as a composite (showing the system's components, also known as white box). The concept of system is generic and is independent of the nature of what is modeled. To help the designer, we give different names to the different kinds of systems: the value 
segment, value network and the company. A value segment is a system made of value networks; a value network is a system made of companies; a company is a system made of employees and IT systems.

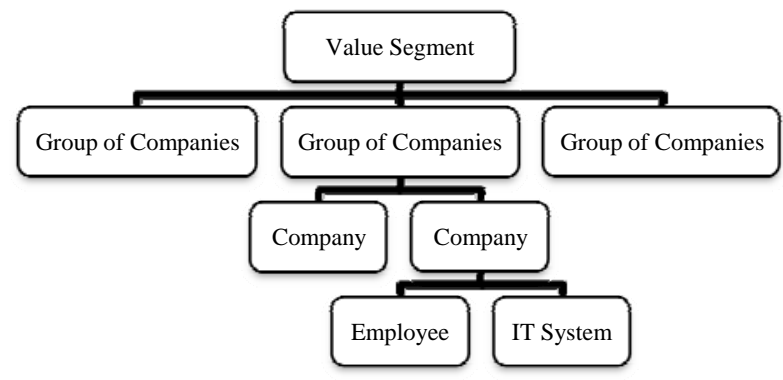

Fig. 2. Hierarchy of systems in enterprise model

A system is either represented as a whole or as a composite along two axes: the organizational axis and functional axis, see Fig. 3. When moving down on the organizational axis, we refine a system into its components. Systems and component systems are represented as block arrows. When moving from left to right on the functional axis, we refine a system's behavior into its component behavior. A behavior is represented as a rounded rectangle. A behavior represents a service offering when it is within a system as a whole. It represents a process when it is within a system as a composite. When moving from the top left corner to the bottom right corner we move from the service offering to the service implementation. This hierarchical representation is only a tool to simplify the conceptualization of a complex reality. We do not imply that the world is hierarchical. However, we can represent reality as a hierarchy in order to simplify the design process, see [8].

In our description of SEAM we make the distinction between us as designers of SEAM and the service provider's project team that uses SEAM for the design of a service. The project team is trans-disciplinary because people of different disciplines design different parts of the service. For example, the service provider's top management and marketing design the strategic value; Experts in finance design the charging system; Marketing experts design the customer value; Experts in logistics design the order fulfillment system.

In all phases of a service design project (Fig. 1), the project team elaborates a single enterprise model for the whole project. The model represents the enterprise and its environment. All changes to the enterprise model are made through the use of diagrams that show different views of the same enterprise model. The two main diagrams are: the system diagram and the supplier/adopter relationship (SAR) diagram. The system diagram represents the hierarchy of systems and the hierarchy of behavior, as shown in Fig. 3. It also includes the building blocks, features and value properties of a service. The SAR diagram represents the relations between these concepts. This paper illustrates the use of these two diagrams use. It does not describe their formal semantics, which is still part of the future work. 


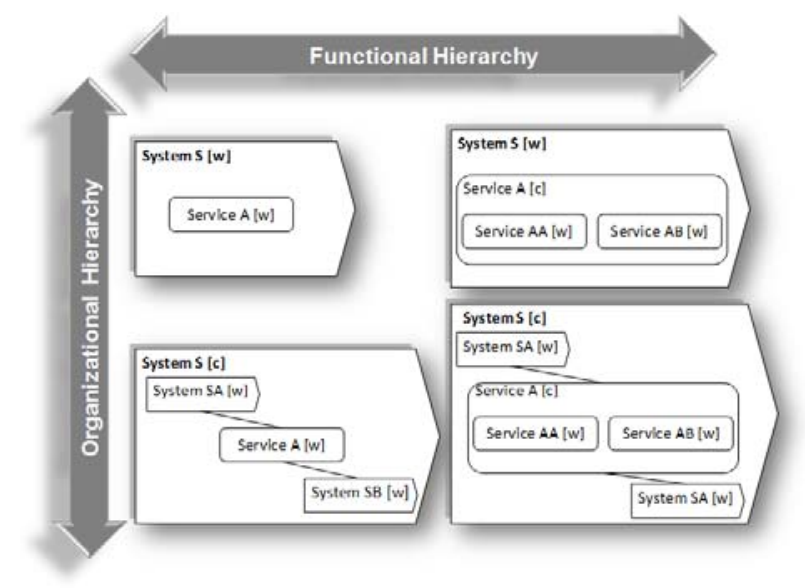

Fig. 3. Double hierarchy in SEAM

We illustrate our method by applying it to the example of service design and implementation at Amazon.com. This example is based on publicly available information about Amazon.com and its market. We describe a hypothetical project, code named A, run by a hypothetical project team, called the A team. Amazon.com was founded as an online bookseller in July 1995. In 1998 Amazon diversified its offering to include new product categories. In November 2000 Amazon introduced the Amazon Marketplace, thereby allowing other companies to place their products alongside Amazon.com's products in the Amazon.com website. Amazon.com is currently offering e-business and IT services to companies of different sizes [9], [10]. In our example, we focus on the transition between selling new books and establishing a partnership with other booksellers to sell used books. We make an as-is model that represents Amazon.com selling new books. We show the misalignment with Amazon.com's future strategy and how the to-be service that enables booksellers to sell used books through Amazon Marketplace might be implemented.

The paper is structured as follows. In section 2, we apply SEAM to the case of service design at Amazon.com. In parallel, we elaborate on the underlying concepts and rationale of our systemic approach. Section 3 includes the related work and in section 4 , we present our future work and the conclusion. 


\section{Designing Service offering and service implementation}

In this Section, we detail each phase of the design process defined in Fig. 1. In each phase, we first present the concepts necessary to make the model, and then we discuss the rationale behind these concepts and illustrate them with the example.

\subsection{Analyzing service offering and implementation as-is}

In this phase, the A team conceptualizes the service offering and the service implementation as-is. As a result, the team members develop a common understanding of the systems that need to be considered in the service description. This information is captured in two system diagrams and in one SAR diagram.

We represent only the Amazon.com Supplier Value Network as a whole and as a composite (Amazon.com Supplier Value Network [w] and [c] in Fig. 4) because we are only interested in the service provided by this value network. When viewing a value network as a whole, we model the service offering. When viewing it as a composite, we model the interactions between the companies that belong to the value network and that participate in the service implementation; in the Amazon.com case, the Publisher Company, the Distributor Company, ISO (ISBIN), the Credit Card Processing Company, Amazon.com Company, Amazon.com's Bank and the Adopter's Bank. In a real project, the A team would also have to analyze the service offered and the service implemented by Amazon.com itself. In that case, the team would have to analyze Amazon.com as a composite as well as a whole. As the design of the service provided by Amazon.com would be done in the same way as the design of the service done by Amazon.com Value Network. We do not present this here.

Amazon.com Supplier Value Network and the SciFi Book Reader Adopter Value Network are components of a value segment (Fig. 4). The supplier value network provides a service to the adopter value network. We usually model the adopter value network as a composite to show all the entities who benefit from the services. In our example, we represent the reader of SciFi books as well as his/her partners. This is useful to analyze the value that the service brings to the SciFi reader (main adopter) as well as to his or her partners, for example the SciFi Community.

In SEAM, we consider the service as an n-to-n relationship: multiple companies participate in the service and multiple entities benefit from the service (directly or indirectly). In SEAM, we also use very concrete examples to make the model more expressive. In consulting, we even write the name of real people and companies. The originality of SEAM is to combine structured diagrams - that capture part of the theory - together with concrete representations of the reality (e.g. real names, photos). This combination brings rigor and relevance to our workshops.

Systems are characterized by their behavior. Understanding system behavior is essential. Systems as wholes and systems as composites have behavior. In service design, we call the behavior of a system as a whole a "service". The behavior of the system as a composite is called a "process". In the system diagram, we identify three processes: the supplier / adopter process (within the value segment as a composite), the process within the adopter value network as a composite and the service 
implementation process within the supplier value network as a composite. Processes are necessary to analyze the interaction between sub-systems. Each sub-system provides services to processes. In the system diagram, we identify two services: the one provided by the supplier value network as a whole and the ones provided by the companies within the supplier value network as composite.

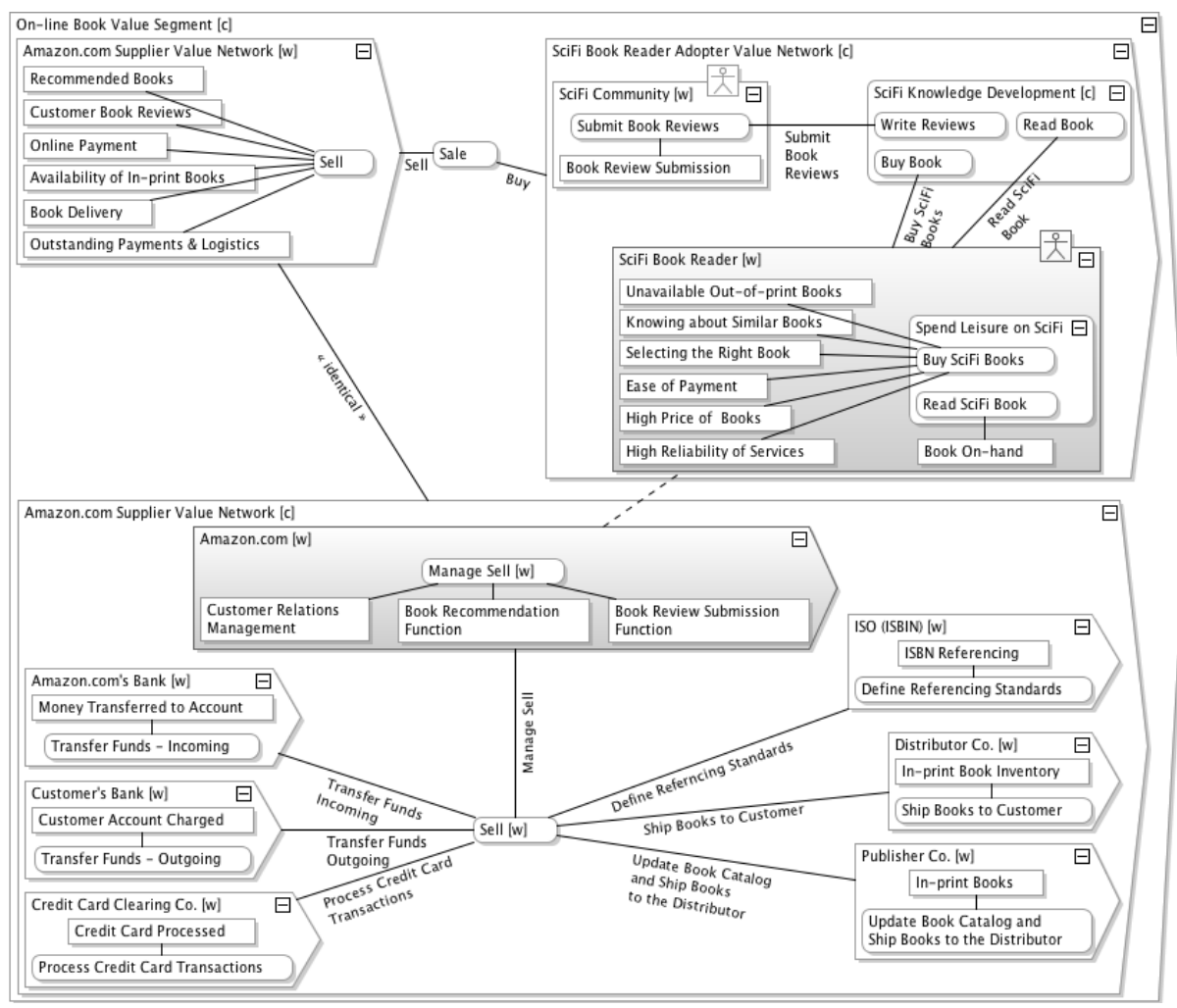

Fig. 4. System diagram, value segment defining the service offering and its implementation asis, process Sell as a whole.

Concretely, in Fig. 4, "Sale" is the process between the Amazon.com Supplier Value Network and the SciFi Book Adopter Value Network. "Sell" within Amazon Supplier Value Network [c] is the implementation process of the service. "Sell" within Amazon Supplier Value Network [w] is the service provided. The "Sell" on the association between the supplier value network and the "Sale" process makes explicit that the "Sell" service is actually related to the process "Sale". The difference between the "Sale" process, the "Sell" service and the "Sell" process is one of the main challenges in designing services. Using a graphical representation greatly facilitates this understanding because it shows the relation between these concepts.

Each system as a whole has properties. Properties are dual to behavior. Properties capture the state of the system. The state is modified by the behavior. When modeling 
services, these properties have different names depending on how they relate to the service offering/implementation. We define three of them:

- building block (property) is a property of a company that implements the service within the supplier value network (as a composite)

- feature (property) is a property of the service provided; it is represented in the supplier value network as a whole (independently of who provide the feature)

- value (property) is a property of the adopter (or of one of its partners); it represents the value the adopter sees in the service. We define the value as an interpretation by a stakeholder of the worth (positive or negative) of a characteristic of a system; the value is expressed in the vocabulary of the stakeholder. In the system diagram, the properties are represented by rectangles. To be able to reason independently on building blocks, features and value properties are useful to understand the viewpoint of the different stakeholders of the services. Value properties are useful to understand customer value. Building blocks are useful to understand the service implementation. Service features are useful to link building blocks and value properties. Finding convincing value properties is a challenge. We have noticed that modeling real customers (such as someone known and who loves SciFi reading) greatly helps identifying customer value.

In Fig. 4, "Book Recommendation Function" and "Book Review Submission Function" are service building blocks provided by Amazon.com within its VN, "Recommended Books" and "Customer Book Reviews" are the service features and, "Selecting the Right Book" and "Knowing about other Similar Books" are property values on the adopter side. These properties are related. For example, the "Selecting the Right Book" value is related to the "Customer Book Reviews" feature, which is supported by the "Book Review Submission Function" building block. The SAR diagram (Fig. 5) makes these relations explicit and specifies the context in which the property exists. So, using a SAR diagram, the A team represents the mapping between the features and the value (service offering design), and between the building blocks and the features (service implementation design). The SAR diagram is an extension of the house of quality [16] in which the relations between "engineering characteristics" and "customer attributes" is established. We added to the house of the quality who provides the characteristics (the "feature" in SEAM and who benefits from the attributes (the "value" in SEAM).

The SAR is also useful to make explicit who provides which building blocks and who benefits from which feature. It is an essential complement to the system diagram as it illustrates who is involved. Sometimes, on the supplier side, we use the RACI technique to capture the involvement of each supplier. RACI stands for Responsible, Accountable, Collaborate, Informed [11]. On the adopter side, we represent with “++”, “+”, “-“ and “- -“ how the value influence the adopters.

The SAR can represent strategies and - more importantly - how features influence strategic value properties. This is done in a similar way as the modeling of the values for the adopters; it is actually a representation of the values for the suppliers. For example, in Fig. 4, the features of the service affect negatively or positively the values of the provider.

The SAR is useful to verify the alignment between all these properties and to identify service improvement opportunities on the basis of the underlying strategic 
Strategic Value

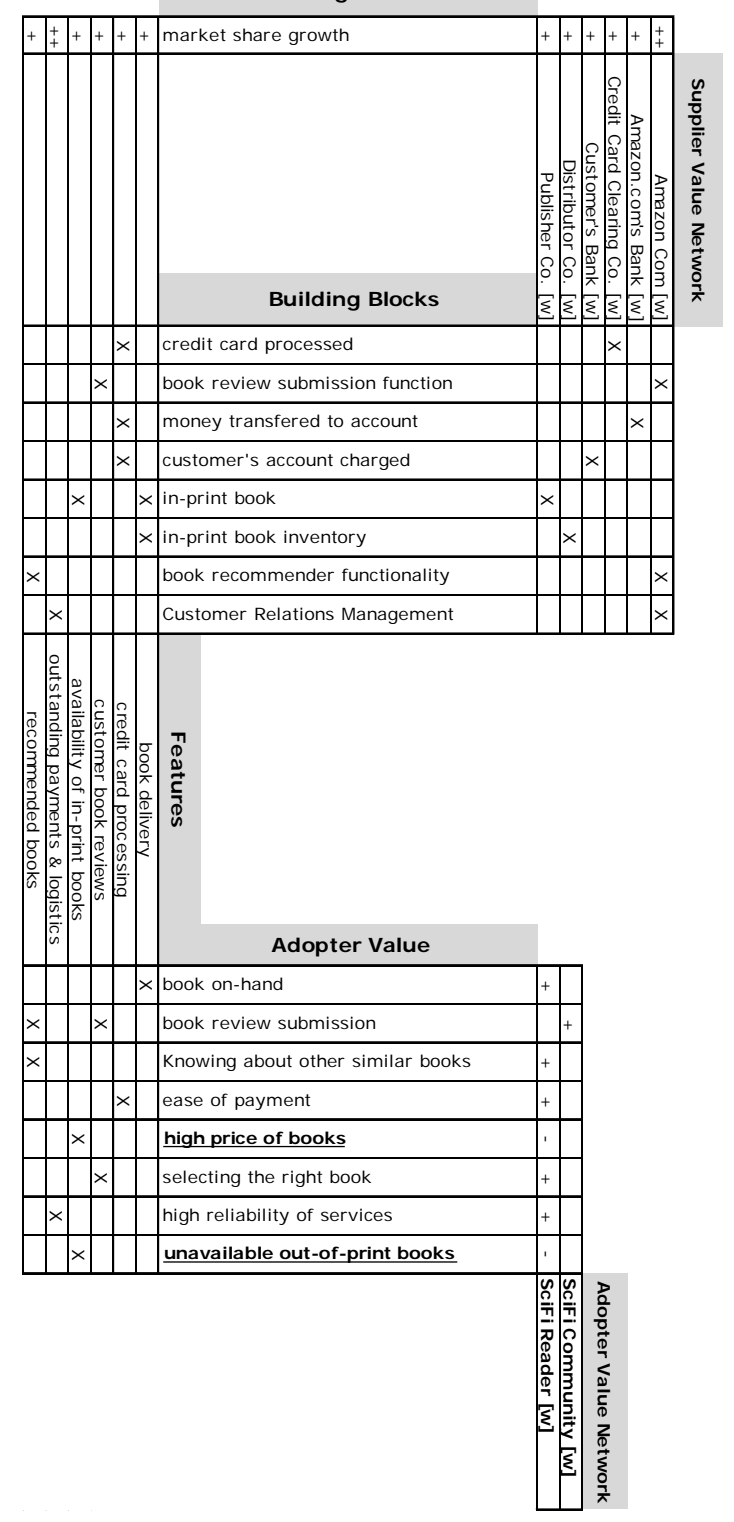

Fig. 5. SAR diagram, service building blocks, features and values as-is. The issues to address are in underline bold.

values of the service. Fig. 5 is a SAR diagram of as-is service building blocks, features and values of Amazon.com. 


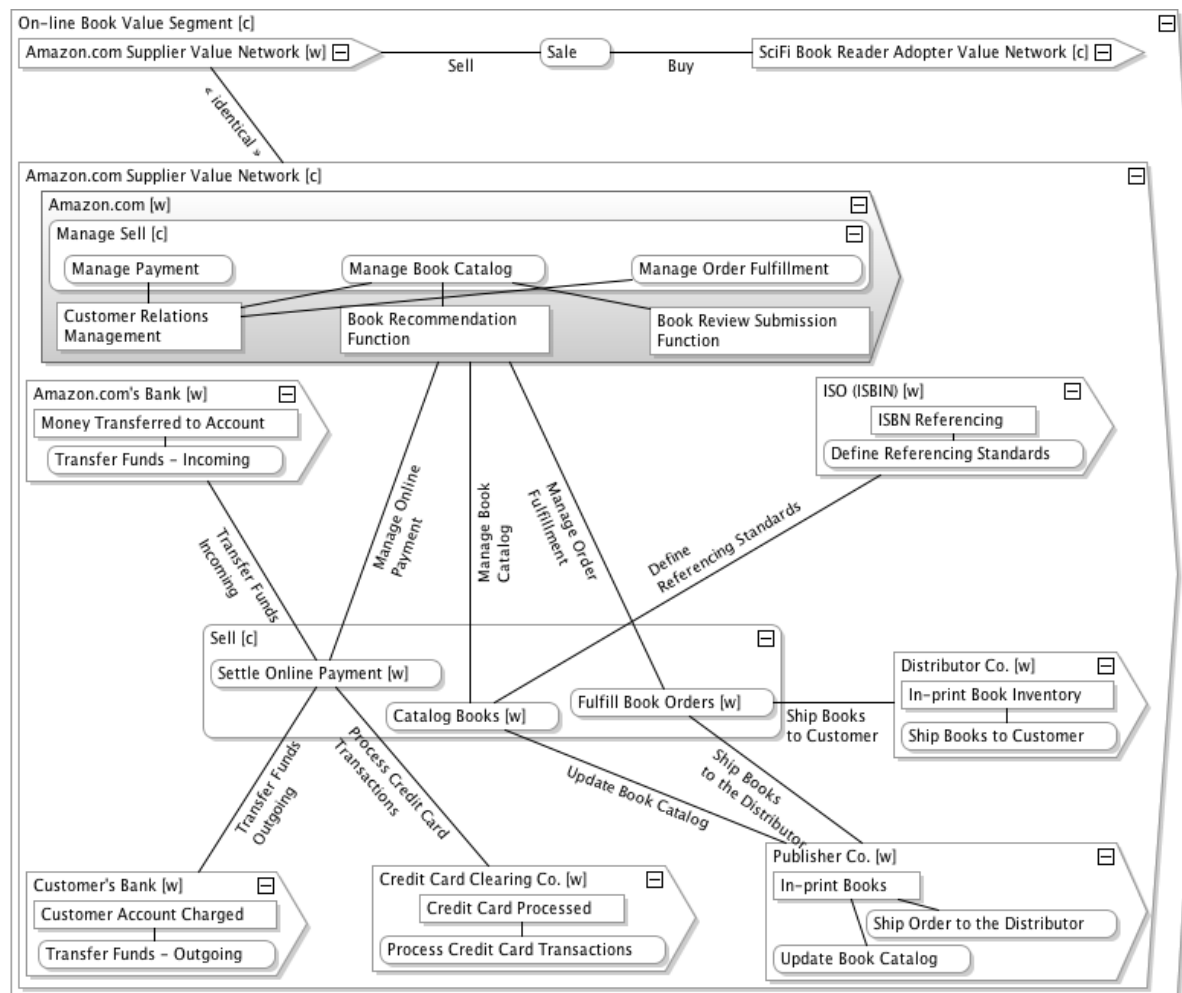

Fig. 6. System diagram, value segment defining the service offering and its implementation as-is, process "Sell" as a composite. This details the responsibilities of each stakeholder.

The system diagram can represent behavior at different levels of detail. This is very useful to separate issues related to the outcome of a behavior as opposed to the detailed execution of the same behavior. For example, in Fig. 4, the "Sell" process is represented as a whole. This means that we only care about what is needed for the process to execute and the outcome of the process. In Fig. 6, we represent the "Sell" process as a composite and the details of the process are then visible. In SEAM, we found it very valuable to work on both representations. In most workshops, the representation of the process as a whole is the adequate one. This representation avoids distracting the workshop participants with implementation details.

\subsection{Identifying improvement opportunities}

In this phase of the service design process we seek improvement opportunities in service offering and service implementation. The goal is to achieve a better alignment between the service and the value properties of the adopters and the strategic value of the suppliers. This is done in three steps: (1) Identification of the issues, (2) listing of the alternatives, and (3) selection of the most appropriate solution. The SAR diagram is used in each step. 
The issues are identified by analyzing the SAR. In the case of Amazon.com, "high price of books" and the "unavailability of books" are the major factors that negatively impact the customer (Fig. 5). Hence, Amazon.com may fail in achieving its strategic objectives and value (i.e. market share growth) unless the current situation is improved.

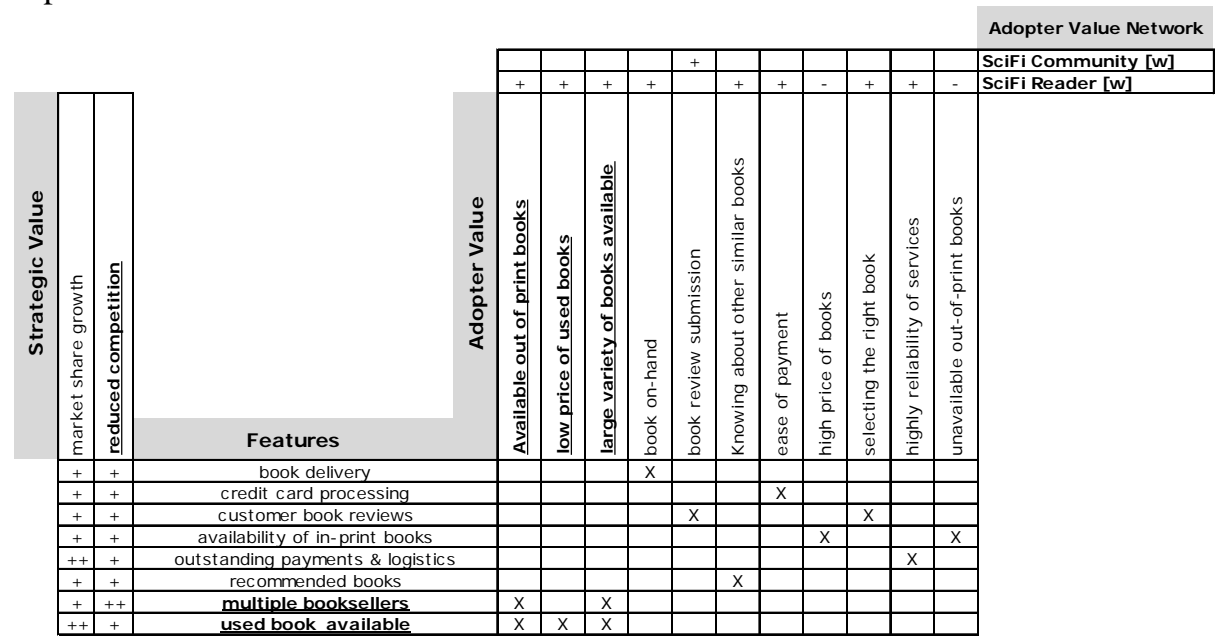

Fig. 7. Proposing service offering to-be. The new value propositions are in underline bold.

Based on these issues, different alternatives can be identified. For example, the high price of the book can be addressed by providing used books, or by providing electronic copies of the book. This last solution might require specific hardware and software solution. The A team needs to evaluate each alternative and to decide which one to develop. This selection process is not detailed in this paper. We make the assumption that providing used book is the selected solution (Fig. 8). Amazon.com offered two new service features to the customer: availability of books from other online booksellers on Amazon.com and selling used books on Amazon.com.

These changes in the service feature, result in creating value on the adopter side such as, providing the customers with "large variety of books", "lower cost of books" and "available out print books" due to the changes in the service offering.

This is also the right time for the A team to think of the strategic value properties of the supplier. For offering these new features, Amazon.com has to cooperate and compete with the booksellers who sell their books alongside Amazon.com books simultaneously. This coopetitive strategy [12] creates the strategic value of "reduced competition" for Amazon.com while leading Amazon.com delivering value to the customers. 


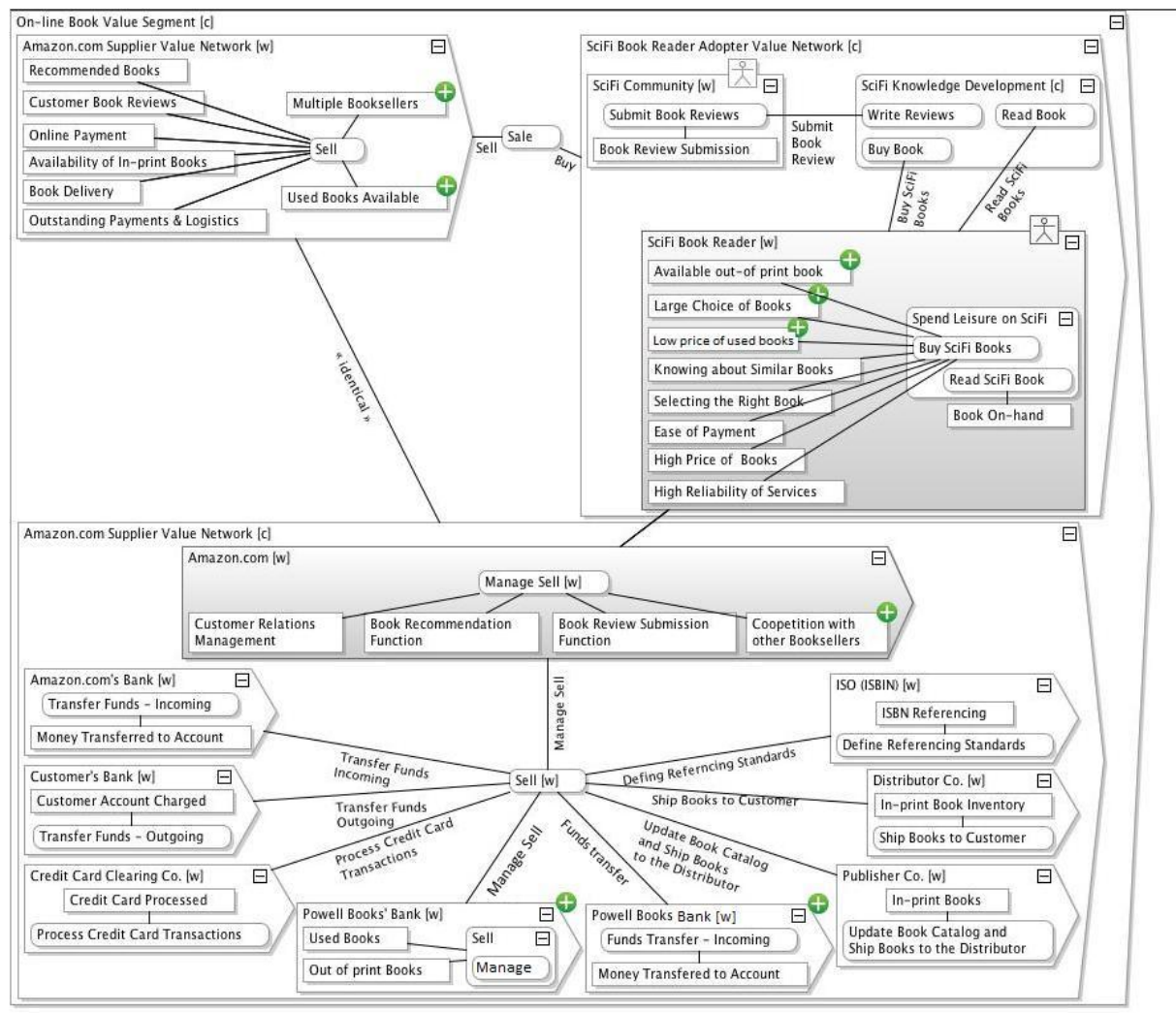

Fig. 8. System diagram, service offering and implementation to-be, process "Sell" as a whole. The additional stakeholders to be considered in the selected solution are marked with a + .

\subsection{Designing the service offering \& implementation to-be}

In this phase, the A team designs the service to-be. In the previous section, the team identified that a new feature is needed: providing used books. It also recognized the need to have multiple book sellers.

The design is done in three steps. First the model is modified to capture the new value properties and features. To add the service building blocks, it is necessary to add the new actors. In our example, Powell Books and the Powell Books's Bank needed to be added. These novelties are represented in the system diagram in Fig. 8. The Sell process is not detailed. Thanks to this, it is not needed to design the detail of the interaction. The model only expresses that two new actors are needed and they will provide the building blocks necessary to provide the features that will lead to the value properties for the customer. At this point the SAR can be regenerated to represent the relations between all the concepts (Fig. 9). 


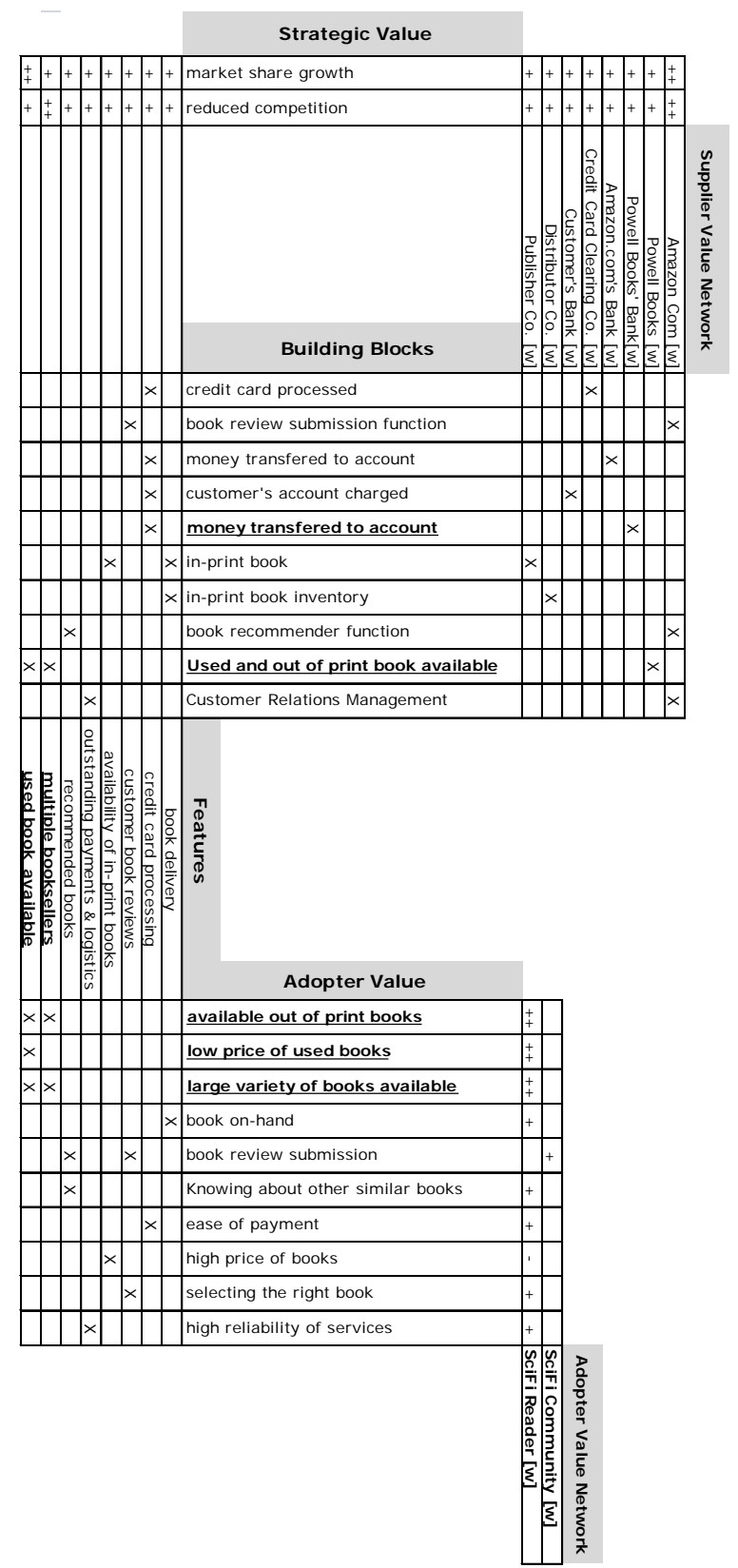

Fig. 9. SAR diagram - Service building blocks, features and values to-be.

To implement the service, the "Sell" process needs to be specified in more detail. In Fig. 8, Sell is defined as a whole and the process outcome is described. The details of the interaction is not described. To refine the process, the A team needs to think in 
terms of instances of behavior. For example, when the catalog of books is created, both Amazon and Powell Books need to participate. The "Catalog Book" action is quite close to the one defined in Fig. 6 with one additional participant. However, the "Fulfill Book Orders" action cannot be modified in the same way. Both Amazon and Powell Books collaborate. In the Fulfill process, Amazon.com and Powell Books compete. There are two processes: one in which Amazon.com provides the books and one in which Powell Books provides the books. The role of the Distributor and the Publisher is especially interesting as they participates in both fulfillment processes. When designing the process, it will be important to analyze how potential conflicts of interests will be solved. Fig. 10 represents the Sell process as a composite.

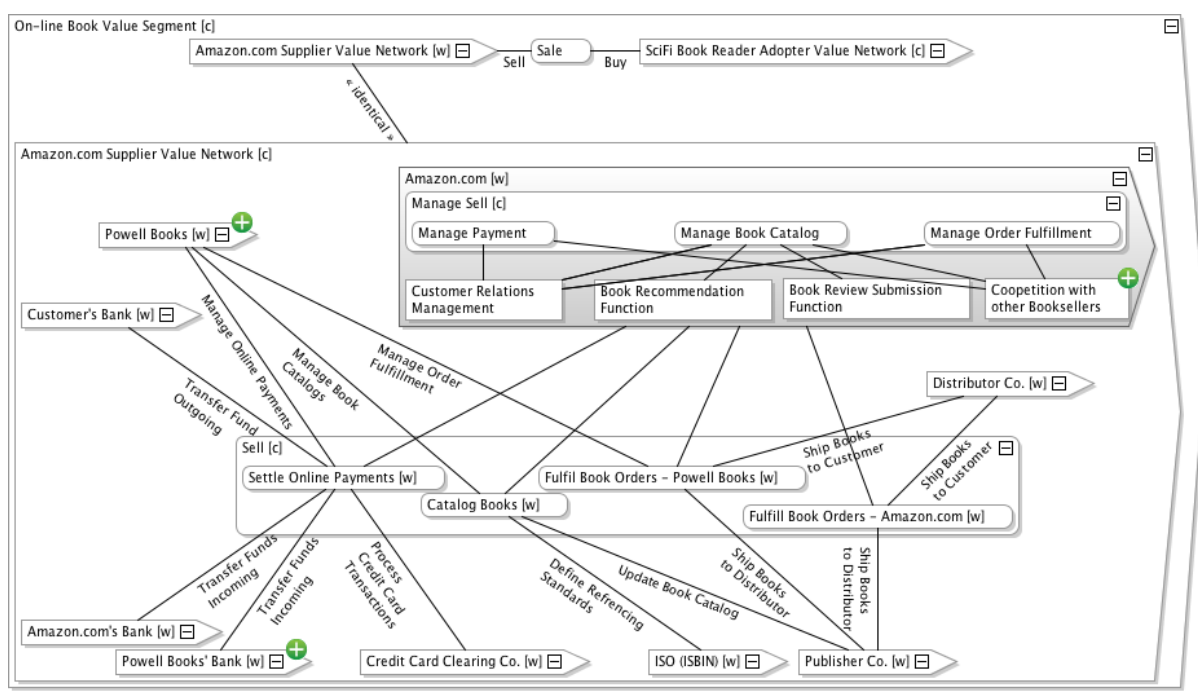

Fig. 10. System diagram, service offering and implementation to-be, process "Sell" as a composite. Definition of the actions in which the new stakeholders participate.

In summary, designing the to-be requires developing a system diagram that represents the project goals (new actors, building blocks, features and value properties). The SAR is then developed to validate the alignment and understand the strategic value properties for the companies in the supplier value network. The system diagram is refined to describe the detail of the business process and to take into considerations issues such as collaborations, conflicts of interest, etc...

\section{Related work}

ITIL [1] is a set of best practices in IT service management which is used by organizations to develop and enhance service management capabilities. The latest version of ITIL, V3, was released in 2007. ITIL V3 focuses on services. It describes service strategy, design, transition, operation and continual service improvement. SEAM a more concrete systemic approach than the ones described in ITIL. 
e3Service [13] is a method for semi-automatically reasoning about matching service offerings with customer needs. In order to make this semi automatic reasoning possible, e3Service assumes that the customer and supplier share the same ontology, that the customer specifies her needs in the same vocabulary as the supplier specifies its offering. In SEAM, we precisely avoid to make this simplifying assumption. This comes at the cost of enormously complicating automatic or event semi-automatic reasoning with the benefit of models that more accurately reflect reality. Also, e3Service defines the value of a service only from the point of view of the customer. In SEAM, this value goes both ways, a service also provides value to its supplier.

$\mathrm{i}^{*}[14]$ is one of the leading modeling method used in the requirements engineering research community for reasoning about FR and NFR. i* provides modeling artifacts for reasoning about alternative satisfactions of NFR. $i^{*}$ models describe relationships as actors dependencies. i* doesn't have an explicit hierarchy or an explicit value constructs. Hence, $i^{*}$ offers better support for reasoning about alternatives . $i^{*}$ has been extended with value reasoning in [15].

House of Quality [16] is an improvement method, which main modeling artifact is very similar to the SEAM SAR model. The House of Quality was derived from Quality Function Deployment, a method that was developed by Japanese companies to improve manufacturing processes for greater customer satisfaction. House of Quality is, therefore, more geared toward manufacturing processes. In addition, the House of Quality does not represent who the providers and the adopters are.

\section{Conclusion and Future Work}

Designing services where the implementation is aligned with the value they provide is a challenging task. It involves people with very different backgrounds (business people to define service offering, financial people for financial model, technical people for implementation). Developing a common enterprise model can help the members of this trans-disciplinary team to agree on what needs to be developed and how to develop it.

In this paper we have shown how to use the SEAM design process and enterprise model for designing services so that their implementation is aligned with the value they provide to both their customers and service providers. The design process has three phases with in which as as-is and a to-be models are built and improvement opportunities are sought. The enterprise model has a double refinement hierarchy that enables us to model a service offering and its implementation simultaneously. We illustrated how to design the service provided by the value network. In a real project, the project team must also design the service provided by the service provider and the service provided by the IT system. This is done using the same diagrams.

The models were developed in a tool, called SeamCAD [17]. In SeamCAD models are built by specifying the changes from one model to the next. It thus maintains the synchronization - as much as possible - between the different models and thus their alignment. Our future work includes validating the approach in commercial workshops, the formalization of the diagrams - and especially of the SAR - and improving SeamCAD. The validation will be done on coopetitive markets and will be based either on a case study or an action research. 


\section{References}

1. ITIL, Version 3. OGC, Office of Government Commerce. London TSO, The Stationery Office (2007)

2. Weinberg, G.M.: An introduction to general systems thinking (silver anniversary ed.). Dorset House Publishing Co., New York, NY, USA (2001)

3. ISO, I.T.U.: ISO/IEC 10746-1, 2, 3, 4| ITU-T Recommendation X. 901, X. 902, X. 903, X. 904.“. Open Distributed Processing-Reference Model 98 (1995)

4. Wegmann, A., Regev, G., De la Cruz, J.D., Lê, L.S., Rychkova, I.: Teaching Enterprise and Service-Oriented Architecture in Practice. In: Second Workshop on Trends in Enterprise Architecture Research (TEAR 2007), St. Gallen, (2007)

5. Wegmann, A., Regev, G., Loison, B.: Business and IT Alignment with SEAM. In: Requirements Engineering for Business Need and IT Alignment Workshop (REBNITA05), Paris (2005)

6. Wegmann, A., Regev, G., Garret, G.-A., Marechal, F.: Specifying Services for ITIL Service Management. In: The International Workshop on Service-Oriented Computing Consequences for Engineering Requirements (SOCCER'08), Barcleona (2008)

7. Rychkova, I., Regev, G., Wegmann, A.: Declarative Specification and Alignment Verification of Services in ITIL. 1st International Workshop on Dynamic and Declarative Business Processes (DDBP 2008), Munich (2008)

8. Checkland, P., Scholes, J.: Soft Systems Methodology in Action. Wiley (1999)

9. Afuah, A., Tucci, C.L.: Internet business models and strategies: Text and cases. McGraw-Hill Higher Education (2000)

10. Spector, R.: Amazon.com: Get Big Fast. Collins Business (2002)

11. A Guide to the Project Management Body of Knowledge. Project Management Institute (2008)

12. Brandenburger, A., Nalebuff, B.: Co-Opetition: the Game Theory That's Changing the Game of Business. Currency (1996)

13. de Kinderen, S., Gordijn, J.: e3 service: A model-based approach for generating needs-driven e-service bundles in a networked enterprise. In: 16th European Conference on Information Systems, Galway (2008)

14. Yu, E.: Towards modelling and reasoning support for early-phase requirements engineering. In: the 3rd IEEE International Symposium on Requirements Engineering (RE'97). Citeseer (1997)

15. Gordijn, J., Yu, E., Van Der Raadt, B.: E-service design using i* and e 3 value modeling. IEEE software 23 , 26-33 (2006)

16. Zachman, J.A.: A framework for information systems architecture. IBM systems journal 38 454-470 (1999)

17. Le, L., Wegmann, A.: SeamCAD: Object-Oriented Modeling Tool for Hierarchical Systems in Enterprise Architecture. Hawaii International Conference on System Sciences 39, 179 (2006) 